\title{
DIAGNÓSTICOS DE ENFERMAGEM MAIS FREQÜENTES EM UMA UNIDADE DE INTERNAÇÃO DE ONCOLOGIA
}

Rosimeire Aparecida Mendes Lopes*

Denise Diniz Macedo** Maria Helena Baena de Moraes Lopes***

LOPES, R. A. M.; MACEDO, D. D.; LOPES, M. H. B. de M. Diagnósticos de enfermagem mais freqüentes em uma unidade de internação de oncologia. Rev.latino-am.enfermagem, Ribeirão Preto, v. 5, n. 4, p. 35-41, outubro 1997.

Este trabalho levanta diagnósticos de enfermagem mais freqüentes, a partir do histórico de enfermagem e procedimento médico, com o objetivo de subsidiar a assistência no Serviço de Enfermagem em Oncologia do Centro de Atenção Integral à Saúde da Mulher (CAISM). Os cinco diagnósticos mais freqüentes foram: potencial para infecção, potencial para temperatura corporal alterada, potencial para aspiração e constipação colônica do padrão trocar, e potencial para intolerância à atividade do padrão mover. O histórico estava direcionado para estes padrões, dificultando a interpretação dos dados que envolviam predominantemente aspectos psicossocioculturais e espirituais, que foram registrados de forma pouco clara ou omitidos.

UNITERMOS: diagnóstico de enfermagem, enfermagem oncológica

\section{INTRODUÇÃO}

O diagnóstico de enfermagem foi introduzido no Brasil em 1967, por Horta, que se baseou na teoria da motivação humana de Maslow. HORTA ${ }^{6}$ propôs uma assistência de enfermagem sistematizada em seis fases, sendo o diagnóstico uma destas fases. Segundo MARIA \& ARCURI ${ }^{8}$, o termo diagnóstico ficou esquecido pela enfermagem brasileira, reaparecendo na literatura a partir de 1988/1989, após uma lacuna de quase 20 anos.

Horta apud CRUZ ${ }^{2}$ em 1977, observou que, na aplicação do processo de enfermagem, o enfermeiro encontra uma grande dificuldade para estabelecer o diagnóstico. Atribuiu como causas: o desconhecimento dos sintomas, das necessidades básicas alteradas e da nomenclatura destas necessidades, entre outras. MARIA $\&$ ARCURI $^{8}$ apontam outros fatores tais como:

- a prática da enfermagem geralmente vinculada ao cumprimento de atividades burocráticas e técnicas, em detrimento do processo de enfermagem.

- ensino de enfermagem que reproduz o modelo biomédico baseado em patologias e execução de técnicas, no qual o aluno vê o processo de enfermagem como um mero exercício acadêmico.

- a resistência das enfermeiras em adotar esse método de trabalho e que acaba por influenciar toda a equipe de enfermagem.

A NANDA (North American Nursing Diagnosis Association) em 1990 definiu o diagnóstico de enfermagem como sendo um julgamento clínico das respostas do indivíduo, ou da família ou da comunidade, aos processos vitais ou aos problemas de saúde atuais ou potenciais, que fornecem a base para a seleção das intervenções de enfermagem para atingir resultados pelos quais o enfermeiro é responsável ${ }^{4}$.

$\mathrm{O}$ uso dos diagnósticos de enfermagem pode trazer benefícios não só para o profissional e cliente, como também para a instituição. Segundo MILLER ${ }^{9}$, para que a enfermeira assista adequadamente o cliente, é necessário conhecer os problemas que ele está experienciando e a falta de clareza na sua identificação implica em perda de tempo e energia, ou mesmo de dinheiro. Para ela o objetivo da enfermagem é prover uma assistência que atenda às necessidades do cliente, enquanto que o da instituição é prestar um serviço efetivo e eficiente. Portanto, o uso dos diagnósticos de enfermagem beneficia a ambos, porque direciona a assistência de enfermagem para as necessidades de cada cliente, facilita a escolha de intervenções mais adequadas, registra de forma objetiva as reações do cliente e permite subsequente avaliação dos cuidados de enfermagem.

* Enfermeira. Assistente Técnica de Direção da Divisão de Enfermagem do CAISM - UNICAMP

** Enfermeira. Supervisora de Enfermagem do Serviço de Enfermagem em Oncologia da Divisão de Enfermagem do CAISM UNICAMP

*** Enfermeira. Professora Assistente Doutora do Dept. de Enfermagem da FCM - UNICAMP e Enfermeira do Programa de Educação Continuada da Divisão de Enfermagem do CAISM - UNICAMP 
$\mathrm{Na}$ literatura, encontramos poucos trabalhos sobre diagnósticos de enfermagem em oncologia. MacAVOY \& MORITZ ${ }^{7}$ identificaram como os diagnósticos mais comuns, em uma população de 48 pacientes oncológicos, a alteração no conforto (dor) e $a$ alteração na nutrição (menos do que o corpo necessita). CHANG et al. ${ }^{1}$ observaram além de dor, risco (ou potencial) para infecção e integridade da pele prejudicada, estudando 59 (32 homens e 27 mulheres) idosos com câncer.

Acreditando na importância do diagnóstico de enfermagem temos, desde 1991, estudado e discutido os diagnósticos segundo a Taxionomia I revisada da NANDA na Divisão de Enfermagem do Centro de Atenção Integral à Saúde da Mulher (CAISM). É meta desta Divisão a capacitação dos enfermeiros no processo e utilização dos diagnósticos de enfermagem.

Mesmo que o Serviço de Enfermagem em Oncologia ainda não tenha implantado todas as etapas da sistematização da assistência de enfermagem, dentre elas o diagnóstico de enfermagem, rotineiramente, as enfermeiras realizam uma entrevista no momento da internação das clientes que serão submetidas à cirurgia. Os dados são coletados em impresso próprio, contendo: histórico, exame físico e tipo de cirurgia.

Foi nosso objetivo neste trabalho levantar os diagnósticos de enfermagem mais freqüentes a partir dos dados registrados nesses impressos. Com base neste estudo, espera-se que as(os) enfermeiras(os) passem a trabalhar com os mais comuns e assim, adquirir habilidade no uso dos diagnósticos de enfermagem.

\section{MÉTODOS}

Foi feito levantamento de 140 prontuários de clientes atendidas no Serviço de Oncologia do CAISM, no período de $1^{\circ}$ de maio a 30 de junho de 1995 (população- alvo). Como critério de seleção, foram incluídos na amostra apenas os prontuários de mulheres que seriam submetidas a cirurgia e que continham o histórico de enfermagem completo, realizado no momento da internação. Desta forma, a população de estudo foi constituída por 30 prontuários.

De acordo com os dados contidos no histórico, que incluia a anamnese e o exame físico realizado pela enfermeira, e o tipo de procedimento médico, foram retrospectivamente levantadas as características definidoras e os fatores de risco e, a partir destes, os diagnósticos de enfermagem. Considerou-se na análise o procedimento médico, uma vez que a terapêutica freqüentemente é um fator de risco para diagnósticos tais como potencial para dor, potencial para infecção e outros.
$\mathrm{Na}$ determinação dos diagnósticos de enfermagem levou-se em conta, ainda: a definição dos diagnósticos, a presença de características definidoras maiores ou de várias características. A identificação dos diagnósticos de enfermagem foi realizada conjuntamente pelas três autoras, com base na Taxionomia I revisada da NANDA com adaptações ao nosso meio conforme NÓBREGA \& GARCIA ${ }^{10}$.

Como o histórico continha dados do momento da admissão da cliente e não estava estruturado de acordo com os padrões de respostas humanas, em muitos casos faltou aprofundamento de informações importantes que permitissem identificar os fatores relacionados. Assim, optou-se por determinar apenas a reação humana e as características definidoras ou fatores de risco.

\section{RESULTADOS E DISCUSSÃO}

A população de estudo compôs-se de mulheres com idade entre 18 e 82 anos (idade média $=50$ anos). A maioria (50\%) era casada, do lar (60\%), com nível básico de escolaridade ( $67 \%$ estudaram até o $1^{\circ}$ grau), de religião predominantemente católica $(63 \%)$.

A distribuição dos procedimentos e cirurgias é apresentada na Tabela 1.

Tabela 1 - Distribuição dos procedimentos e cirurgias

\begin{tabular}{lc}
\hline PROCEDIMENTO/CIRURGIA & FREQÜÊNCIA \\
& $\mathbf{n}$ \\
\hline Mastectomia e axilectomia & 1 \\
Mastectomia com reconstrução & 2 \\
Quadrantectomia e axilectomia & 4 \\
Exérese de nódulo & 5 \\
Exérese de ductos & 1 \\
Ressecção ampla de mama & 2 \\
Laparotomia & 3 \\
Pan-histerectomia total abdominal & 1 \\
Histerectomia total abdominal & 1 \\
Conização e curetagem uterina & 5 \\
Exérese de colo restante & 1 \\
Curetagem uterina de prova & 1 \\
Vulvectomia radical & 1 \\
Ampliação de margem de vulva e & 1 \\
linfadenectomia pélvica & \\
Braquiterapia de cúpula vaginal & 1 \\
ToTAL & $\mathbf{3 0}$ \\
\hline
\end{tabular}

Na Tabela 2 são apresentadas as freqüências dos diagnósticos, características definidoras e fatores de risco do padrão de respostas humanas TROCAR. Os 
diagnósticos mais freqüentes foram: potencial para infecção (presente em todos os casos), potencial para temperatura corporal alterada (18 casos), potencial para aspiração (11 casos), constipação colônica (oito casos), potencial para proteção alterada (sete casos) e potencial para déficit do volume de líqüido (seis casos).

Tabela 2 - Freqüência de diagnósticos de enfermagem, características definidoras e fatores de risco do padrão trocar

\begin{tabular}{|c|c|c|c|}
\hline DIAGNÓSTICO & $\begin{array}{c}\text { FREQÜÊNCIA } \\
\mathbf{n}\end{array}$ & $\begin{array}{c}\text { CARACTERISTICA DEFINIDORA } \\
\text { FATOR DE RISCO }\end{array}$ & $\begin{array}{l}\text { FREQÜÊNCIA } \\
\mathbf{n}\end{array}$ \\
\hline $\begin{array}{l}\text { Potencial para infecção } \\
\text { Potencial para temperatura } \\
\text { corporal alterada }\end{array}$ & $\begin{array}{l}30 \\
18\end{array}$ & $\begin{array}{l}\text { procedimento invasivo (cirurgia) } \\
\text { uso de anestésico (sedação) } \\
\text { extremo de idade }\end{array}$ & $\begin{array}{c}30 \\
17 \\
4\end{array}$ \\
\hline Potencial para aspiração & 11 & $\begin{array}{l}\text { diminuição do nível de consciência (efeito } \\
\text { da anestesia) }\end{array}$ & 11 \\
\hline Constipação colônica & 8 & $\begin{array}{l}\text { fezes secas e endurecidas } \\
\text { freqüência reduzida } \\
\text { esforço para evacuar }\end{array}$ & $\begin{array}{l}8 \\
3 \\
1\end{array}$ \\
\hline Potencial para proteção alterada & 7 & $\begin{array}{l}\text { tratamento oncológio em curso (cirurgia, } \\
\text { radio ou quimioterapia prévia) } \\
\text { excessiva perda por vias normais (vômito) }\end{array}$ & $\begin{array}{l}7 \\
2\end{array}$ \\
\hline $\begin{array}{l}\text { Potencial para deficit do volume } \\
\text { de líquido }\end{array}$ & 6 & $\begin{array}{l}\text { perda de líquidos através de vias anormais } \\
\text { (dreno) } \\
\text { medicação (diurético) }\end{array}$ & $\begin{array}{l}2 \\
4\end{array}$ \\
\hline Eliminação urinária alterada & 4 & disúria & 4 \\
\hline $\begin{array}{l}\text { Nutrição alterada: ingestão maior } \\
\text { do que as necessidades corporais }\end{array}$ & 2 & $\begin{array}{l}\text { peso acima do ideal para a idade, sexo, } \\
\text { altura e estrutura }\end{array}$ & 2 \\
\hline $\begin{array}{l}\text { Nutrição alterada: ingestão } \\
\text { menor do que as necessidades } \\
\text { corporais }\end{array}$ & 1 & inabilidade percebida para ingerir alimento & 1 \\
\hline Integridade da pele prejudicada & 1 & $\begin{array}{l}\text { solução de continuidade da pele } \\
\text { (deiscência) }\end{array}$ & 1 \\
\hline Integridade tissular prejudicada & 1 & tecido lesado & 1 \\
\hline Constipação & 1 & $\begin{array}{l}\text { freqüência de eliminação menor do que o } \\
\text { padrão usual }\end{array}$ & 1 \\
\hline Constipação percebida & 1 & uso de laxante & 1 \\
\hline TOTAL & 87 & & 96 \\
\hline
\end{tabular}


Os diagnósticos, características definidoras e fatores de risco do padrão RELACIONAR são apresentados na Tabela 3. Os diagnósticos deste padrão tiveram baixa frequiência (quatro casos). $\mathrm{O}$ diagnóstico disfunção sexual foi identificado em apenas dois casos. Contudo, provavelmente esta freqüência esteja subestimada, uma vez que os dados do histórico de enfermagem eram incompletos pela falta de aprofundamento das questões relacionadas à sexualidade. Outro aspecto relevante é que o histórico foi realizado apenas no momento da admissão. Caso os dados fossem coletados no período pós-operatório, outros diagnósticos de enfermagem poderiam ser identificados.

A perda de uma parte do corpo, como a mama, está estreitamente relacionada à sexualidade, à autoimagem e à auto-estima. Uma doença como o câncer, que é debilitante e requer tratamento prolongado, pode levar a uma alteração no processo familiar, considerandose que a clientela atendida na instituição é predominantemente feminina e responsável pela manutenção do lar, quer como donas de casa, quer como trabalhadoras.

Tabela 3 - Freqüiência de diagnósticos de enfermagem, características definidoras e fatores de risco do padrão relacionar

\begin{tabular}{lclc}
\hline \multicolumn{1}{c}{ DIAGNÓSTICO } & $\begin{array}{c}\text { FREQÜÊNCIA } \\
\mathbf{n}\end{array}$ & $\begin{array}{l}\text { CARACTERISTICA DEFINIDORA } \\
\text { FATOR DE RISCO }\end{array}$ & $\begin{array}{c}\text { FREQÜÊNCIA } \\
\mathbf{n}\end{array}$ \\
\hline $\begin{array}{l}\text { Processo familiar } \\
\text { alterado }\end{array}$ & 2 & $\begin{array}{l}\text { inabilidade dos membros da familia para } \\
\text { relacionarem-se entre si e para propiciar } \\
\text { crescimento e amadurecimento mútuos }\end{array}$ & 2 \\
Disfunção sexual & 2 & $\begin{array}{l}\text { verbalização do problema (dor, "falta de } \\
\text { vontade") }\end{array}$ & 2 \\
TOTAL & $\mathbf{4}$ & & $\mathbf{4}$ \\
\hline
\end{tabular}

Na Tabela 4 são apresentados os diagnósticos, características definidoras e fatores de risco do padrão MOVER. O diagnóstico mais frequiente deste padrão foi potencial para intolerância à atividade (oito casos).
O diagnóstico de mobilidade física prejudicada foi identificado em apenas uma mulher que já era mastectomizada unilateralmente. Caso o histórico fosse realizado após a cirurgia, a freqüência desse diagnóstico seria provavelmente maior.

Tabela 4 - Freqüência dos diagnósticos de enfermagem, características definidoras e fatores de risco do padrão mover

\begin{tabular}{lclc}
\hline \multicolumn{1}{c}{ DIAGNÓSTICO } & $\begin{array}{c}\text { FREQÜÊNCIA } \\
\mathbf{n}\end{array}$ & $\begin{array}{l}\text { CARACTERISTICA DEFINIDORA } \\
\text { FATOR DE RISCO }\end{array}$ & $\begin{array}{c}\text { FREQÜÊNCIA } \\
\mathbf{n}\end{array}$ \\
\hline $\begin{array}{l}\text { Potencial para mobilidade } \\
\text { fisica prejudicada }\end{array}$ & 8 & $\begin{array}{l}\text { submissão a procedimento médico que requer } \\
\text { restrição de movimentos (linfadenectomia) }\end{array}$ & 8 \\
$\begin{array}{l}\text { Mobilidade física } \\
\text { prejudicada }\end{array}$ & 1 & $\begin{array}{l}\text { restrição de movimentos imposta por prescrição } \\
\text { médica }\end{array}$ & 1 \\
$\begin{array}{l}\text { Manutenção da saúde } \\
\text { alterada }\end{array}$ & 1 & $\begin{array}{l}\text { desinteresse expresso em comportamentos de } \\
\text { promoção da saúde }\end{array}$ & 1 \\
\begin{tabular}{l} 
ToTAL \\
\hline
\end{tabular} & 10 & & $\mathbf{1 0}$ \\
\hline
\end{tabular}

As freqüências dos diagnósticos de enfermagem, características definidoras e fatores de risco do padrão
PERCEBER são apresentadas na Tabela 5. Os diagnósticos deste padrão foram identificados em apenas dois casos. 
Tabela 5 - Frequiência dos diagnósticos de enfermagem, características definidoras e fatores de risco do padrão perceber

\begin{tabular}{lclc}
\hline \multicolumn{1}{c}{ DIAGNÓSTICO } & $\begin{array}{c}\text { FREQÜENNCIA } \\
\mathbf{n}\end{array}$ & $\begin{array}{l}\text { CARACTERISTICA DEFINIDORA } \\
\text { FATOR DE RISCO }\end{array}$ & $\begin{array}{c}\text { FREQÜENCIA } \\
\mathbf{n}\end{array}$ \\
\hline Desesperança & 1 & $\begin{array}{l}\text { pas sividade } \\
\text { falta de inciativa }\end{array}$ & 1 \\
& 1 & mudança relatada da acuidade auditiva & 1 \\
$\begin{array}{l}\text { Sensopercepção alterada } \\
\text { (auditiva) }\end{array}$ & & & 1 \\
TOTAL & 2 & & 3 \\
\hline
\end{tabular}

Quanto ao padrão CONHECER, o exame de mama) foi identificado em quatro casos diagnóstico de deficit de conhecimento (sobre o auto- $\quad$ (Tabela 6).

Tabela 6 - Freqüiência dos diagnósticos de enfermagem, características definidoras e fatores de risco do padrão conhecer

\begin{tabular}{lclc}
\hline DIAGNÓSTICO & $\begin{array}{c}\text { FREQÜENCIA } \\
\mathbf{n}\end{array}$ & $\begin{array}{l}\text { CARACTERISTICA DEFINIDORA } \\
\text { FATOR DE RISCO }\end{array}$ & $\begin{array}{c}\text { FREQ̈̈ENCIA } \\
\mathbf{n}\end{array}$ \\
\hline $\begin{array}{l}\text { Deficit de conhecimento } \\
\text { (sobre o auto-exame de } \\
\text { mama) }\end{array}$ & 4 & verbalização do problema & 4 \\
\hline
\end{tabular}

Na Tabela 7 são apresentadas as freqüências dos diagnósticos de enfermagem, características definidoras e fatores de risco do padrão SENTIR. O diagnóstico medo foi encontrado em três casos. O objeto do medo identificado pela cliente relacionava-se à anestesia e à possibilidade de detectar câncer na mama através do autoexame.

Tabela 7 - Freqüência dos diagnósticos de enfermagem, características definidoras e fatores de risco do padrão sentir

\begin{tabular}{lclc}
\hline DIAGNósTICO & $\begin{array}{c}\text { FREQÜENCIA } \\
\mathbf{n}\end{array}$ & \multicolumn{1}{c}{$\begin{array}{c}\text { CARACTERISTICA DEFINIDORA } \\
\text { FATOR DE RISCO }\end{array}$} & $\begin{array}{c}\text { FREQÜENCIA } \\
\mathbf{n}\end{array}$ \\
\hline Medo & 3 & $\begin{array}{l}\text { habilidade para identific ar o objeto do me do } \\
\text { (anestesia, câncer mamário) }\end{array}$ & 3 \\
& 2 & comportamento de fuga & 1 \\
Cor & 1 & $\begin{array}{l}\text { relato verbal de dor experimentada por mais de } \\
\text { seis meses }\end{array}$ & 1 \\
Dor crônica & & & 7 \\
TOTAL & 6 & & \\
\hline
\end{tabular}

\section{CONSIDERAÇÕES FINAIS}

O diagnóstico abre possibilidades para o desenvolvimento da enfermagem porque cria uma linguagem própria para descrever os problemas do cliente que a(o) enfermeira(o) tem competência para resolver. 
Na opinião de $\mathrm{CRUZ}^{3}$, a busca e a organização dos conhecimentos para a proposição de ações independentes da(o) enfermeira(o) ficam prejudicadas quando não se tem uma terminologia que expresse o julgamento que fazemos acerca dos problemas dos clientes. Assim, o uso de uma linguagem própria de enfermagem contribui para o desenvolvimento do seu corpo de conhecimentos, além de efetivar a comunicação entre as(os) enfermeiras(os). Segundo HERBERTH \& GOSNELL ${ }^{5}$, o uso dos diagnósticos de enfermagem contribuiriam para a melhor definição da prática clínica dentro da enfermagem oncológica, por se tratar de uma especialidade complexa.

Mundinger apud HERBERTH \& GOSNELL ${ }^{5}$ observou que a documentação das respostas do cliente e do grau de sucesso das medidas de enfermagem implementadas para resolver respostas que comprometem o seu estado de saúde poderiam indicar ações independentes da(o) enfermeira(o) que incrementam a habilidade em lidar com situações e a sobrevida do cliente com câncer.

A análise de dados a partir de um referencial de enfermagem é um processo árduo, ao mesmo tempo é uma oportunidade de aprendizagem e de crescimento pessoal.

Como era de se esperar, devido a nossa formação embasada no modelo biomédico, os diagnósticos de enfermagem do padrão trocar e mover foram identificados com maior freqüência. Esse fato deve-se a coleta de dados, que estava melhor direcionada para estes padrões de respostas humanas, e a dificuldade de interpretação dos achados que envolviam predominantemente aspectos psicossocioculturais e espirituais, pela falta de clareza ou insuficiência de dados.
O presente trabalho apresenta a limitação por tratar-se de um estudo baseado em dados de prontuário analisados retrospectivamente e que, por esta razão, não puderam ser melhor validados ou complementados. Outro aspecto relevante foi o fato do histórico ter sido realizado somente no momento da admissão. Os dados assim coletados não permitem planejar adequadamente a assistência de enfermagem porque a coleta de dados é um processo contínuo e as respostas das clientes modificam-se de acordo com as experiências vivenciadas durante o período de internação.

Com exceção das que já tinham diagnóstico de câncer no momento da internação, as mulheres do estudo apresentavam diagnósticos característicos de uma clientela geral e não de clientes oncológicos. Caso os dados analisados fossem referentes a clientes clínicos, o perfil dos diagnósticos provavelmente seria outro, já que este grupo inclui aqueles fora de possibilidade terapêutica médica os quais necessitam de um maior número de intervenções e de cuidados de enfermagem.

Esse estudo permitiu identificar algumas lacunas da taxionomia dos diagnósticos de enfermagem. Embora através da Taxionomia I revisada da NANDA não tenha sido possível identificar características definidoras indicadoras do diagnóstico de perfusão tissular alterada, as clientes submetidas à mastectomia com reconstrução apresentam alto risco para este diagnóstico devido ao enxerto (mama reconstruída a partir da pele e tecido subcutâneo proveniente da região abdominal). Um diagnóstico não contemplado na taxionomia e que está relacionado com o procedimento cirúrgico é o potencial para dor, cujas intervenções de enfermagem devem se dar em nível de prevenção da dor.

Pretendemos em uma próxima etapa complementar este trabalho com a realização de estudos prospectivos.

\section{THE MOST FREQUENT NURSING DIAGNOSES IN AN ONCOLOGY ADMISSION UNIT}

The aim of this paper was to survey the most frequent nursing diagnoses by means of the nursing history and medical procedure in order to help the care provided at the Oncology Nursing Service of Women Health Whole Care (Centro de Atenção Integral à Saúde da Mulher - CAISM). The five most common diagnoses were: risk for infection, risk for altered body temperature, risk for aspiration and colonic constipation of the exchanging pattern, and risk for activity intolerance of the moving pattern. Data collection was based on these patterns. Data predominantly involving social, cultural, psychological and spiritual aspects were hardly interpreted, because they were poorly registered or omitted.

KEY WORDS: nursing diagnosis, oncologic nursing

\section{DIAGNÓSTICOS DE ENFERMERIA MAS FRECUENTES EN UNA UNIDADE DE HOSPITALIZACIÓN DE ONCOLOGÍA}

El presente trabajo levanta diagnósticos de enfermería más frecuentes, a partir de la historia realizada por enfermería y del procedimiento médico, con el objetivo de ayudar la asistencia en el Servicio de Enfermería en Oncologia del Centro de 
Atención Integral a la Salud de la Mujer (CAISM). Los cinco diagnósticos más frequentes fueron: potencial para infección, potencial para temperatura corporal alterada, potencial para aspiración y constipación colónica de patrón cambiar y potencial para intolerancia a la actividad del patrón mover. La historia estaba direccionada para estos patrones, dificultando la interpretación de los datos que predominantemente envuelven aspectos psico-socioculturales y espirituales, los que fueron registrados de forma poco clara u omitidos.

TÉRMINOS CLAVES: diagnóstico de enfermería, enfermería oncológica

\section{REFERÊNCIAS BIBLIOGRÁFICAS}

01. CHANG, B. L.; VREDEVOE, D.; HIRSH, M. Allergy as risk factor for nursing care problems in the elderly cancer patient. Cancer Nurs., v. 18, n. 2, p. 83 - 88, Apr. 1995.

02. CRUZ, I. C. F. da Diagnóstico de enfermagem e sua aplicação: revisão da literatura.Rev. Esc.Enfermagem USP, v. 24, n. 1, p. 149 - 162, abr. 1990.

03. CRUZ, D. de A.L.M. da Os diagnósticos de enfermagem no ensino e na pesquisa. Rev. Esc. Enfermagem USP, v.26, n.3, p. 427-434, dez. 1992.

04. FARIAS, J. N. de et al. Diagnóstico de enfermagem: uma abordagem conceitual e prática. João Pessoa: Santa Marta, 1990. $160 \mathrm{p}$.

05. HERBERTH, L.; GOSNELL, D. J. Nursing diagnosis for oncology nursing practice. Cancer Nurs., v. 10, n. 1, p. 41-51, 1987.
06. HORTA, W. de A. Histórico. In: . Processo de enfermagem. São Paulo: EPU, 1979. p. 37 38.

07. MacAVOY, S.; MORITZ, D. Nursing diagnoses in an oncology population. Cancer Nurs., v. 15, n. 4, p. 264 - 270, Aug. 1992.

08. MARIA, V. L. R. ; ARCURI, E. A. de M. O ensino e a prática do diagnóstico de enfermagem em uma instituição governamental. In: SIMPÓSIO NACIONAL SOBRE DIAGNÓSTICOS DE ENFERMAGEM, 1, São Paulo, 1991. Anais. São Paulo: GIDE - SP, 1991. p. 6 - 45.

09. MILLER, E. How to make nursing diagnosis work: administrative and clinical strategies. Norwalk/ San Mateo: Appleton \& Lange, 1989. 366 p.

10. NÓBREGA, M. M. L.; GARCIA, T.R. Uniformização da linguagem dos diagnósticos de enfermagem da NANDA: sistematização das propostas do II SNDE. João Pessoa: A União, 1994. $138 \mathrm{p}$. 